\title{
Corticosteroid-associated Changes Resembling a Demyelinating Brain Lesion in Diffuse Large B-cell Lymphoma (DLBCL): A Case Report
}

\author{
Gio Earnest de la Cruz and Justine Alessandra Uy \\ Department of Laboratory Medicine and Pathology, The Medical City, Pasig City, Philippines
}

\section{ABSTRACT}

Biopsies of suspected lymphoma cases with history of pre-biopsy corticosteroid therapy present several diagnostic issues, such as the inability to demonstrate the neoplastic hematolymphoid cells, the similarity of post-corticosteroid changes with inflammatory demyelinating lesions, and the possibility of a demyelinating lesion preceding a central nervous system lymphoma. This report presents the case of a 51-year-old immunocompetent male with a solitary callosal mass, with immunomorphologic features suggestive of a demyelinating lesion on initial biopsy, and upon re-biopsy after three months revealed a diffuse large B-cell lymphoma. Awareness of these issues in post-corticosteroid stereotactic biopsy specimens, together with adequate clinical and radiologic data, is important for proper diagnosis and further therapeutic guidance.

Key words: corticosteroids, demyelination, diffuse large B cell, lymphoma

ISSN 2507-8364 (Online)

Printed in the Philippines.

Copyright $\odot 2021$ by the PJP.

Received: 1 August 2021.

Accepted: 23 August 2021.

Published online first: 7 September 2021.

https://doi.org/10.21141/PJP.2021.14

Corresponding author: Gio Earnest D. de la Cruz, MD, MBA

E-mail: gioeddelacruz@gmail.com

ORCiD: https://orcid.org/0000-0003-2589-850X

\section{INTRODUCTION}

Diffuse large B-cell lymphoma (DLBCL) is a neoplasm of medium to large B lymphoid cells, commonly presenting among elderly males in the seventh decade. Primary central nervous system lymphoma (PCNSL) accounts for $<1 \%$ of all non-Hodgkin lymphomas and 2.4-3\% of all brain tumors. The most common locations are the frontal lobe, periventricular brain parenchyma, and the posterior fossa. Corpus callosum involvement is rare and is reported in only $5 \%$ of cases. ${ }^{1}$ Secondary involvement of the central nervous system (CNS) by systemic lymphoma has an estimated incidence of $2-5 \%,,^{2,3}$ and leptomeningeal is more common than parenchymal metastasis. ${ }^{4}$

The gold standard for diagnosis is histopathologic examination of stereotactic biopsy specimens. However, in cases wherein corticosteroid treatment was initiated prior to biopsy, typical microscopic characteristics of DLBCL may not be seen. Features may be non-specific or show features of demyelinating lesions. Furthermore, demyelinating disease may confound the diagnosis of DLBCL because of their similar radiologic characteristics and response to corticosteroids.

This report discusses a case of a callosal mass that was initially suggestive on histopathology to exhibit demyelinating disease, but on second biopsy of the mass three months after, was diagnosed as DLBCL.

\section{CASE}

A 51-year-old male presented with a six-month history of intermittent bifrontal headache and progressive memory lapses. On admission, the patient was given two doses of Dexamethasone $4 \mathrm{mg}$, with slight improvement of symptoms. Brain MRI with contrast revealed an enhancing 
well-defined mass at the corpus callosum, extending to the occipital forceps, with mass effect. The mass was T1Whypointense and T2W-intermediate/mildly hyperintense, and radiologic considerations included a CNS lymphoma.

Stereotactic biopsy of the lesion was performed seven months after symptom onset. This was one week from the administration of the last corticosteroid dose. Immunohistomorphologic evaluation revealed a lesion composed of many macrophages and reactive astrocytes, as seen in Figure 1. Few scattered parenchymal and perivascular inflammatory cells were also present, mostly composed of T-cells, and few B-cells highlighted by CD20 and PAX5. These cells, although arranged around vessels, had small nuclei and inconspicuous nucleoli. The lesion also demonstrated a low proliferation index. The case was signed out as a macrophage-rich lesion with chronic inflammation, astrogliosis, and axonopathy, with considerations primarily favoring a tumefactive demyelinating disease. With the history of presurgical corticosteroid therapy, and the clinical and radiologic suspicion of a CNS lymphoma, included in the report was a suggestion for a re-biopsy at least three weeks after the last steroid administration. On admission, further workup revealed a firm mass on the left submandibular area. Aspiration was performed, and results were consistent with cyst contents.

In the interim, the patient continued to have memory lapses, and developed new-onset left-sided weakness and disorientation. Subsequent imaging studies performed in another institution revealed interval increase in the size of the mass with significant vasogenic edema. A second stereotactic biopsy of the lesion was done ten
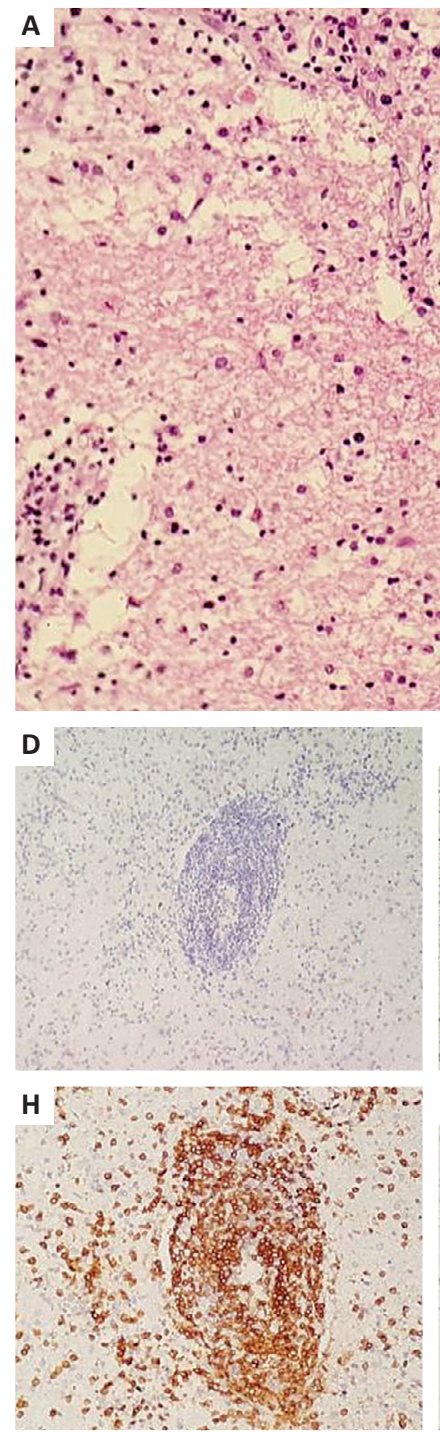
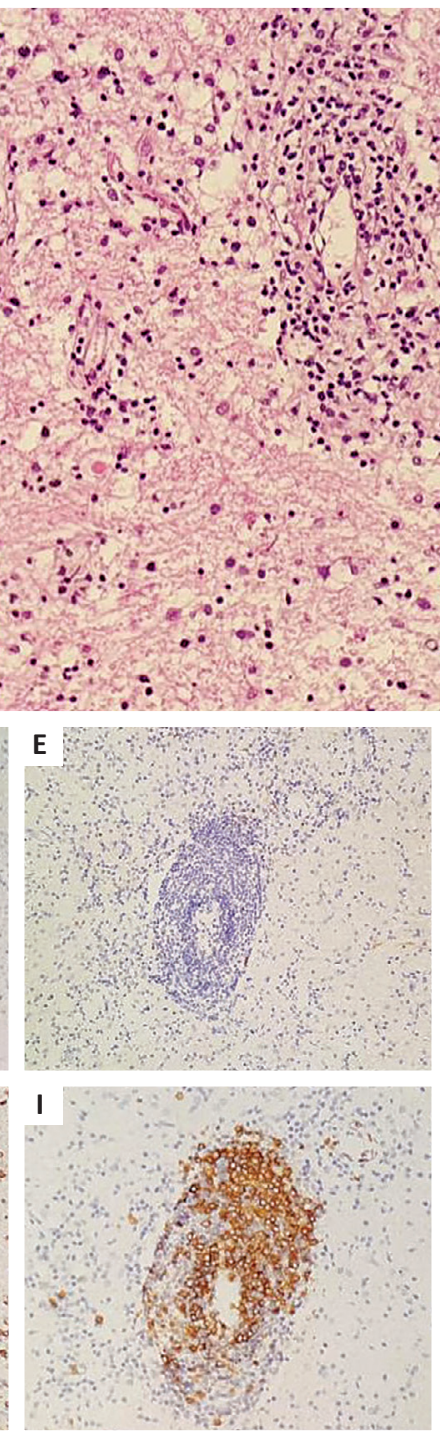
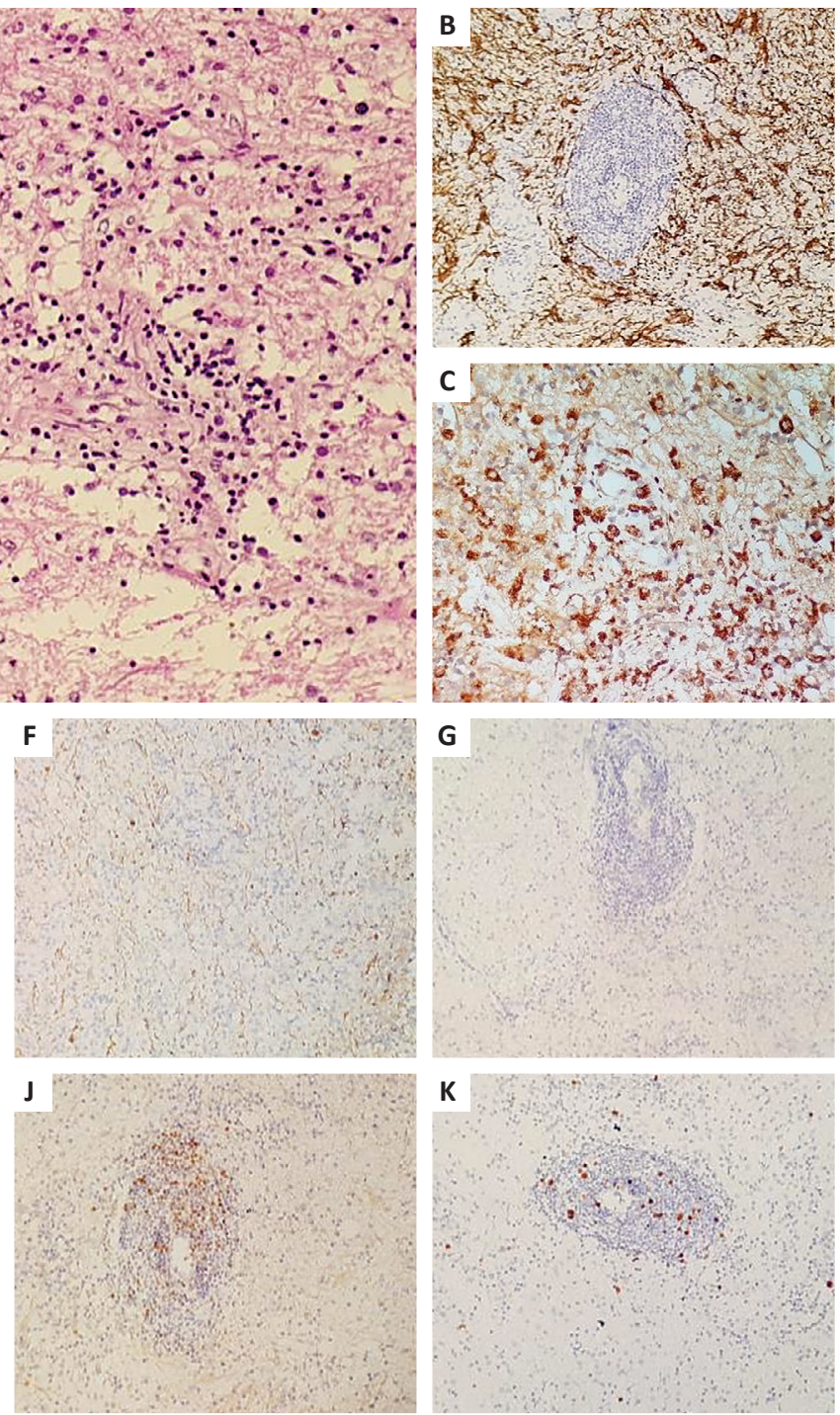
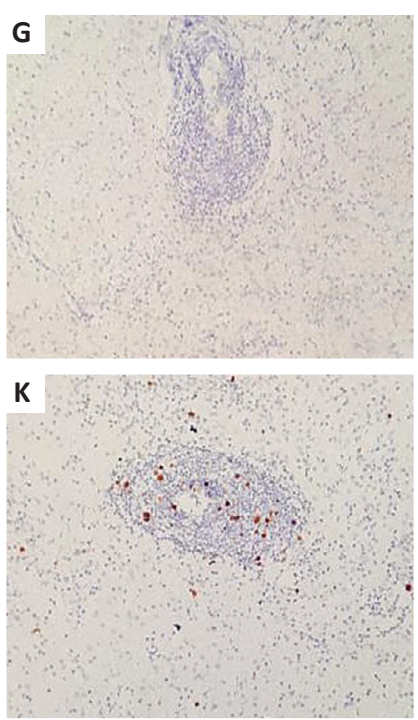

Figure 1. Morphology of the first biopsy. (A) Neural tissue with scattered inflammatory cells, some of which surround vessels (H\&E, $\mathrm{x} 200$ ). (B) Immunohistochemistry for glial fibrillary acidic protein (GFAP) demonstrates astrogliosis, as it highlights the reactive astrocytes (x200). (C) CD68 highlights numerous parenchymal and perivascular macrophages (x200). (D) p16 and (E) p53 staining are positive in few scattered reactive astrocytes, expected in wild-type genotypes (x200). (F) Neurofilament highlights relatively intact axonal processes. (G) Immunohistochemistry for IDH R132H expression is negative (x200). (H) CD3 is positive in perivascular and scattered parenchymal T-cells, (I) CD20 and (J) PAX5 highlight few small parenchymal and perivascular B-cells, and (K) Ki-67 is positive in $3-5 \%$ of perivascular cells, and in less than $1 \%$ of glial cells (x200). 
months from the symptom onset, corresponding to three months from the last corticosteroid dose. The biopsy now showed sheets of enlarged atypical cells with round to oval, hyperchromatic nuclei, and scant cytoplasm, as seen in Figure 2. Immunohistochemistry studies with CD20 revealed diffuse strong positivity in the enlarged cells, supporting a B-cell neoplasm. Further studies with Cyclin D1 and Bcl-2 ruled out other considerations such as a mantle cell lymphoma and marginal zone lymphoma, and studies with CD10, MUM1, Bcl-6, c-MYC confirmed the diagnosis of a diffuse large B-cell lymphoma, nongerminal center B-cell subtype.

Further workup included serologic tests for HIV, HAV, $\mathrm{HBV}$, and HCV - all of which were negative. A positron emission tomography-computed tomography (PET-CT) was also done, which revealed uptake in one cervical lymph node, correlating to the previously biopsied left submandibular area. Post- operative period was uneventful.

\section{DISCUSSION}

PCNSL is a rare extranodal form of lymphoma, accounting for $1 \%$ of non-Hodgkin lymphomas and 3-5\% of primary brain tumors in immunocompetent patients, with a peak incidence at the fifth to seventh decade of life, and a median age of 56 years. It is more common among males, with a male-to-female ratio of 3:2. The most common locations include the posterior fossa (13\%), frontal lobe $(8 \%)$, parietal lobe $(7 \%)$, occipital lobe $(3 \%)$, basal ganglia and periventricular brain parenchyma (10\%), and corpus callosum (5\%). ${ }^{1}$ Secondary CNS involvement by systemic lymphomas is more common than PCNSL; reported incidence is at around 5\% but range from 2-27\%. This wide variation is attributed to patient inclusion criteria, different histologic subtypes, and different methods for assessing CNS involvement. ${ }^{2}$ DLBCL is the most common systemic lymphoma to involve the CNS, at an estimated incidence of $2-5 \% .^{3}$ Leptomeningeal $(33-100 \%)$ is more
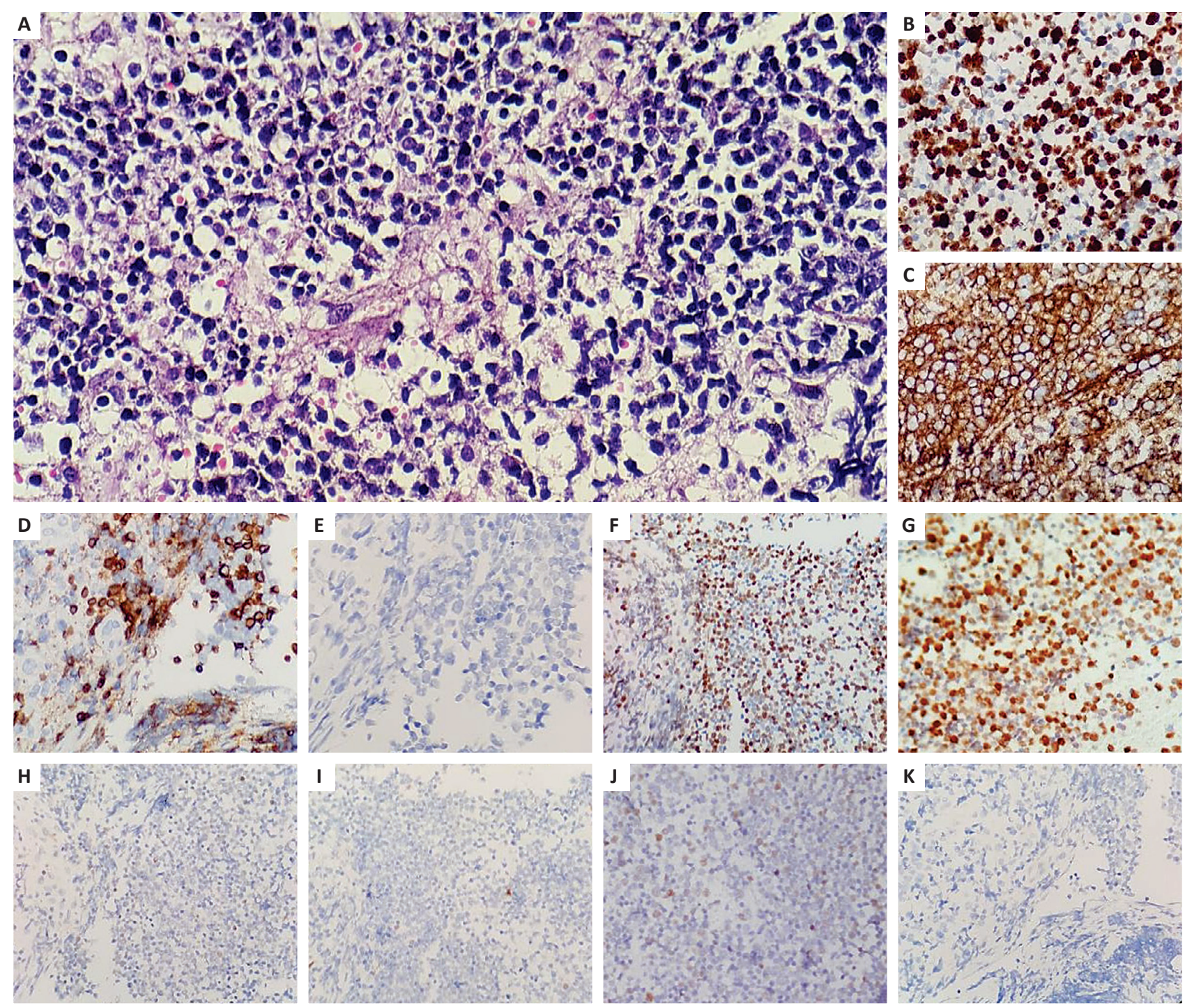

Figure 2. Morphology of the second biopsy. (A) Microsections show sheets of medium to large lymphoid cells with hyperchromatic nuclei, irregular nuclear borders, occasionally prominent nucleoli, and scant cytoplasm (H\&E, x400). (B) Ki-67 shows a high proliferation index (greater than 90\%), and (C) CD20 highlights the neoplastic B-cells (x200); (D) Immunohistochemistry for CD3 is positive in scattered reactive T-cells, and (E) CD30 is negative (x200); (F) Bcl-6 and (G) MUM-1 are positive in approximately $70 \%$ of the cells; (H) Bcl-2, (I) Cyclin D1, (J) c-myc and (K) CD10 are negative in the cells of interest (x200). 
common in cases of secondary CNS involvement, and occasionally may involve the superficial cortex. ${ }^{4}$ Deep, periventricular involvement typically seen in PCNSL is rare in secondary lymphomas. ${ }^{2}$ CNS involvement of systemic lymphomas usually manifests within two years of diagnosis of initial diagnosis, with a median time of less than one year. ${ }^{3}$ Patients would present with cognitive dysfunction, psychomotor slowing, and focal neurologic symptoms. ${ }^{1}$

MRI is a sensitive technique for detecting DLBCL, which will demonstrate hypointense T1-weighted and isointense to hyperintense T2-weighted images, with densely enhancing postcontrast images, and perilesional edema. Although these findings were consistent with the radiologic profile of the patient's callosal mass, stereotactic biopsy is still necessary, as it is considered the gold standard for diagnosis. ${ }^{2,3}$

On microscopy, the tumor is typically highly cellular, containing large areas of geographic necrosis centrally, and perivascular cuffing and splitting of the argyrophilic fiber network peripherally. Clusters or individual tumor cells infiltrate the surrounding tissue diffusely, accompanied by a prominent astrocytic and microglial activation and a reactive inflammatory infiltrate consisting of mature $T$ and B cells and foamy histiocytes. The tumor cells have atypical medium- to large-sized round, oval, irregular, or pleomorphic nuclei with distinct nucleoli, expressing mature B-cell markers (PAX5, CD19, CD20, CD22, and CD79a) without plasma cell markers (CD38 and CD138). Most cases also express BCL6 and IRF4/MUM1. CD10 is a marker that is more common in systemic DLBCL than in primary CNS lymphoma. ${ }^{5}$ Distinction of secondary CNS involvement of systemic DLBCL cannot be distinguished morphologically from PCNSL. Molecular genetics such as comparative IG gene analysis of both tumors is necessary to differentiate between these entities. ${ }^{3}$

In some cases of CNS neoplasms, corticosteroid therapy is initiated to lower increased intracranial pressure. However, steroids induce apoptosis in malignant and even non-malignant lymphocytes, which can lead to rapid disappearance of the lesion on imaging and within biopsy specimens. ${ }^{3}$ The diagnosis of CNS lymphoma, then, becomes challenging when biopsies are taken postcorticosteroid therapy. The microscopic picture can show nonspecific reactive and chronic inflammatory lesions consisting of $\mathrm{T}$ cells, macrophages, and reactive astrocytes. Reactive gliosis and microglial activation may be prominent. Necrosis may or may not be present. Foamy macrophages may also predominate, and their presence raises the suspicion of steroid-mitigated CNS lymphoma. In some cases, few neoplastic B cells may be present only in small numbers. Mature B-cells may be mingled, usually forming perivascular collections, or scattered individually throughout the parenchyma. ${ }^{2,3}$

Demyelinating lesions, primarily multiple sclerosis (MS), may also confound the diagnosis of DLBCL, because of their similar radiologic features on MRI and their responsiveness to steroid therapy. ${ }^{2}$ MS plaques that appear on MRI as tumor-forming may be biopsied and misdiagnosed as glioma or lymphoma in as often as $18 \%$ of cases. MS plaques would typically appear on MRI as multiple, well- demarcated homogeneous small ovoid lesions with no mass effect. However, atypical radiologic features may confound the diagnosis, such as a solitary large lesion larger than $2 \mathrm{~cm}$, associated mass effect, perilesional edema, and presence of ring enhancement. Active MS plaques are classically characterized by preferential loss of myelin relative to axons; prominent perivascular lymphocytic infiltrates composed mostly of T-cells, and to a lesser extent B-cells; large numbers of parenchymal and perivascular macrophages; reactive astrocytosis; and cerebral edema. ${ }^{6}$ Although the diagnosis may be straightforward based on morphology, problematic patterns include specimens which only show a few lymphoma cells in a background of reactive brain tissue, and active demyelinating lesions with proliferating perivascular B cells. ${ }^{2}$ The morphologic features of demyelinating lesions may even be indistinguishable from steroid-induced inflammatory reactions, in the absence of evidence for demyelination. ${ }^{3}$ However, subtle histologic features that are more prominent in steroidtreated lymphomas than in primary demyelination include the presence of incomplete and inhomogeneous demyelination, extensive inflammation - particularly of CD3 + T lymphocytes, ${ }^{7}$ and decreased axon density and preservation, and the presence of axonal spheroids. ${ }^{8}$ In addition, other clinical and radiologic findings may raise the suspicion of PCNSL, such as: middle to older age with no prior clinical episodes or radiographic lesions suggestive of MS, lack of spinal cord involvement, and increased enhancement or lesion size over time ${ }^{9}-$ features present in our case.

Another entity that poses a diagnostic dilemma are contrast-enhancing "sentinel" demyelinating lesions that precede PCNSL. These lesions are radiologically and morphologically like MS and are followed by development of PCNSL within 12 months but may be up to two years. ${ }^{1}$ Possible explanations for this include malignant transformation of a chronic inflammatory process, demyelination due to anti-myelin bodies secreted by lymphoma cells, and sampling error. ${ }^{2}$ Another theory posed is that this reaction may represent the first immune response against the developing PCNSL. ${ }^{3}$ These lesions regress spontaneously or upon corticosteroid treatment. There is no published report on the incidence of this entity; however, there are a few case reports that describe this phenomenon ${ }^{9-11}$ and none describe this entity in the local literature. On microscopy, these lesions will show no neoplastic B cells, and instead would exhibit variable demyelination, $\mathrm{T}$ and $\mathrm{B}$ cells, plasma cells, macrophages, astrogliosis, and well-preserved axons.

The effects of corticosteroids on CNS lymphoma are temporary, and recurrence may occur once therapy is ceased. Because of this, prompt diagnosis may be delayed. In a study by Bruck et al., of 933 patients, pretreatment with corticosteroids of patients suspected with PCNSL prevented diagnosis in up to $50 \% .^{12}$ Another study found that steroid use increased the risk of vanishing tumor among PCNSL cases by $14 \%$ per $100 \mathrm{mg}$ dexamethasone equivalent, leading to delayed diagnosis. ${ }^{13}$ These provide credence to the recommendations in current practice to withhold corticosteroid use in those suspected with CNS lymphomas prior to stereotactic biopsy. The rate of re- 
biopsy among PCNSL patients with pre-biopsy corticosteroid treatment was at $12 \%$ in one retrospective study. ${ }^{14}$

The dose and timing of steroid administration prior to a non-diagnostic biopsy have wide ranges: In a Turkish study of PCNSL patients, the patients received $4 \mathrm{mg}$ dexamethasone with 6-hour intervals, ranging from 2-30 days, with an interval between steroid use and biopsy of 0-2 days. $53 \%$ of these patients needed a second biopsy or PCR to establish the diagnosis of PCNSL. ${ }^{15}$ In the study of vanishing tumor among PCNSL cases, the mean cumulative dose in dexamethasone equivalents was $555+$ $704 \mathrm{mg}$ prior to the nondiagnostic biopsy. ${ }^{13}$ Finally, in the retrospective study mentioned previously, the cases had a median total dosage of steroids equivalent to $325 \mathrm{mg}$ (range $25-6325 \mathrm{mg}$ ), taken at a median duration of 5 days (range 1-90 days), and with median time from treatment to biopsy of 0 days (range $0-180) .{ }^{14}$ Our case received a total of $8 \mathrm{mg}$ of dexamethasone, taken within two days, the last dose of which was given eight days prior to the biopsy. This total dose is below the median and mean total steroid doses reported in other studies that led to a non-diagnostic stereotactic biopsy, further showing the wide variability to responses in corticosteroids among CNS lymphoma patients.

The recommended duration of corticosteroid abstinence has not been established, mostly because of high individual variation of response. In line with this, even a repeat biopsy cannot guarantee a definitive diagnosis. ${ }^{3}$ In previous studies, the interval between the first and second biopsy ranged from six to eleven months. ${ }^{9,10}$ In a cohort study by Barrantes-Freer et al., the interval between the first and second biopsy for which PCNSL was histologically confirmed was 3 to 32 weeks. ${ }^{7}$ In another study, the median time to recurrence after the first non-diagnostic biopsy was 53 days, but with a wide range from 8 to 648 days. ${ }^{13}$ Our case was diagnosed three months after the last dose, which is consistent with the previous studies cited.

\section{CONCLUSION}

The diagnosis of CNS lymphoma becomes a challenge once corticosteroid therapy has been initiated, as it induces apoptosis of the neoplastic B cells and leads to rapid regression of the tumor on imaging and within biopsies. The morphologic features of post-steroid biopsies may not demonstrate the neoplastic B cells, and may only exhibit non-specific inflammatory and reactive processes indistinguishable from other demyelinating diseases such as MS. As such, corticosteroid therapy for cases of suspected CNS lymphoma should be withheld to prevent delay of diagnosis and to initiate the proper therapy regimen. However, it should be kept in mind that repeat biopsies even after withholding corticosteroid therapy may not guarantee a proper diagnosis of CNS lymphoma. Furthermore, demyelinating lesions like MS may precede PCNSL and further complicates the proper diagnosis of a CNS lymphoma.

\section{ETHICAL CONSIDERATIONS}

This case report was submitted to and acknowledged by the Institutional Review Board of The Medical City.

\section{STATEMENT OF AUTHORSHIP}

Both authors certified fulfillment of ICMJE authorship criteria.

\section{AUTHOR DISCLOSURE}

Both authors declare no conflict of interest.

\section{FUNDING SOURCE}

None.

\section{REFERENCES}

1. Deckert M, Paulus W, Kluin PM, Ferry JA. Lymphomas. In: Louis DN, Ohgaki H, Wiestier OD, et al. WHO classification of tumours of the central nervous system. Lyon: International Agency for Research on Cancer (IARC); 2016.

2. Paulus W, Perry A. Lymphomas and histiocytic tumors. In: Perry A, Brat DJ. Practical Surgical Neuropathology: A diagnostic approach. Philadelphia: Elsevier; 2010.

3. Deckert M. Lymphomas and Haemopoietic Neoplasms. In: Love S, Perry A, Ironside J, Budka H. Greenfield's Neuropathology, 9th ed. USA: CRC Press; 2015.

4. Patrij K, Reiser M, Wätzel L, et al. Isolated central nervous system relapse of systemic lymphoma (SCNSL): clinical features and outcome of a retrospective analysis. Ger Med Sci. 2011; 9:Doc11. PMID: 21698087. PMCID: PMC31 18693. https://doi. org//10.3205/000134.

5. Gascoyne RD, Campo E, Jaffe ES, et al. Diffuse large B- cell lymphoma, NOS. In: Swerdlow SH, Campo E, Harris NL, et al. WHO classification of tumors of hematopoietic and lymphoid tissues. Lyon: International Agency for Research on Cancer (IARC); 2017.

6. Schmidt RE. White matter and myelin disorders. In: Perry A, Brat DJ. Practical surgical neuropathology: a diagnostic approach. Philadelphia: Elsevier; 2010.

7. Barrantes-Freer A, Engel AS, Rodriguez-Víllagra OA, et al. Diagnostic red flags: steroid-treated malignant CNS lymphoma mimicking autoimmune inflammatory demyelination. Brain Pathol. 2018; 28(2):225-33. PMID: 28213912. PMCID: PMC8028373. https://doi. org/10.1111/bpa.12496.

8. Giannini C, Dogan A, Salomão D. CNS Lymphoma: a practical diagnostic approach. J Neuropathol Exp Neurol. 2014;73(6):478-94. PMID: 24806301. https:// doi.org/10.1097/NEN.0000000000000076.

9. Kvarta MD, Sharma D, Castellani RJ, et al. Demyelination as a harbinger of lymphoma: a case report and review of primary central nervous system lymphoma preceded by multifocal sentinel demyelination. BMC Neurol. 2016;16:72. PMID: 27206499. PMCID: PMC4875602. https://doi.org/ 10.1186/s12883-016-0596-1.

10. Alderson L, Fetell MR, Sisti M, Hochberg F, Cohen M, Louis DN. Sentinel lesions of primary CNS lymphoma. J Neurol Neurosurg Psychiatry. 1996;60(1):102-5. PMID: 8558135. PMCID: PMC486201. https://doi. org/10.1136/jnnp.60.1.102. 
11. Husseini L, Saleh A, Reifenberger G, Hartung H, Kieseier BC. Inflammatory demyelinating brain lesions heralding primary CNS lymphoma. Can J Neurol Sci. 2012;39(1):6-10. PMID: 22384490. https://doi.org/10.1017/s0317167100012610.

12. Brück W, Brunn A, Klapper W, et al. Differential diagnosis of lymphoid infiltrates in the central nervous system: experience of the network lymphomas and lymphomatoid lesions in the nervous system. Pathologe. 2013;34(3):186-97. PMID: 23471726. https://doi.org/10.1007/s00292-013-1742-9.

13. Yim E, Umemura Y, Sun Y, Junck L. EPID-27. The vanishing tumor phenomenon in the diagnosis of primary CNS lymphoma. Neuro-Oncol. 2020;22(2): ii84. https://doi.org/10.1093/neuonc/noaa215.345
14. Porter AB, Giannini C, Kaufmann T, et al. Primary central nervous system lymphoma can be histologically diagnosed after previous corticosteroid use: a pilot study to determine whether corticosteroids prevent the diagnosis of primary central nervous system lymphoma. Ann Neurol. 2008;63(5):662-7. PMID: 18481293. https://doi.org/10.1002/ana.21366.

15. Önder E, Arikök AT, Önder S, et al. Corticosteroid pretreated primary CNS lymphoma: a detailed analysis of stereotactic biopsy findings and consideration of interobserver variability. Int J Clin Exp Pathol. 2015;8(7):7798-808. PMID: 26339344. PMCID: PMC4555672.

Disclaimer: This journal is OPEN ACCESS, providing immediate access to its content on the principle that making research freely available to the public supports a greater global exchange of knowledge. As a requirement for submission to the PJP, all authors have accomplished an AUTHOR FORM, which declares that the ICMJE criteria for authorship have been met by each author listed, that the article represents original material, has not been published, accepted for publication in other journals, or concurrently submitted to other journals, and that all funding and conflicts of interest have been declared. Consent forms have been secured for the publication of information about patients or cases; otherwise, authors have declared that all means have been exhausted for securing consent.

\section{Publish in the new PJP. Visit our website: htp://philippinejournalofpathology.org}

